\title{
Biomarker and pathway analyses of urine metabolomics in dairy cows when corn stover replaces alfalfa hay
}

\author{
Huizeng Sun, Bing Wang, Jiakun Wang, Hongyun Liu and Jianxin Liu ${ }^{*}$
}

\begin{abstract}
Background: Alfalfa hay and corn stover are different type of forages which can significantly impact a cow's lactation performance, but the underlying metabolic mechanism has been poorly studied. We used biomarker and pathway analyses to characterize related biomarkers and pathways based on urine metabolomics data from different forage treatments. Urine was collected from 16 multiparous Holstein dairy cows fed alfalfa hay (AH, high-quality forage, $n=8$ ) and corn stover (CS, low-quality forage, $n=8$ ) respectively. Gas chromatography-time of flight/mass spectrometry (GC-TOF/MS) was performed to identify metabolites in urine and the metaboanalyst online platform was used to do biomarker and pathway analysis.

Results: Hippuric acid (HUA) and N-methyl-glutamic (NML-Glu) indicated the most significant difference between the two diets, when statistically validated by biomarker analysis. HUA was also validated by standard compound quantitative method and showed significant higher concentration in CS group than AH group (2.8282 vs. $0.0005 \mathrm{mg} / \mathrm{mL} ; P<0.01)$. The significant negative correlation between milk yield and HUA $\left(R^{2}=0.459 ; P<0.01\right)$ and significant positive correlation between milk yield and NML-Glu $\left(R^{2}=0.652 ; P<0.01\right)$ were characterized. The pathway analysis revealed that these different metabolites were involved in 17 pathways including 7 influential pathways (pathway impact value $>0$ ): Tyr metabolism, starch and sucrose metabolism, amino sugar and nucleotide sugar metabolism, galactose metabolism, Phe, Tyr and Try biosynthesis, purine metabolism, and glycerolipid metabolism. Based on the metabolome view map, the Phe, Tyr and Try biosynthesis pathway exhibited the highest impact value (0.50), and the Holm-Bonferroni multiple testing-based analysis revealed the most significant difference in the Tyr metabolism pathway ( $\mathrm{Holm} P=0.048$ ).

Conclusions: The identified HUA and NML-Glu may serve as potential biomarkers for discriminating CS and AH diets and could be used as candidates for milk yield related mechanistic investigations. Integrated network pathways associated with related metabolites provide a helpful perspective for discovering the effectiveness of forage quality in lactation performance and provides novel insights into developing strategies for better utilization of CS and other low-quality forage in China.
\end{abstract}

Keywords: Biomarker, Dairy cow, Metabolomics, Pathway, Urine

\section{Background}

Forage quality greatly affects dairy cow performance $[1,2]$. Using traditional nutritional methods, it is difficult to discern metabolic alterations and characterize alterations in key metabolic pathways when dairy cows are fed diets differing in forage quality. Metabolomics is an effective means to explain the overall complex and essential

\footnotetext{
* Correspondence: liujx@zju.edu.cn
Institute of Dairy Science, MoE Key Laboratory of Molecular Animal Nutrition,
College of Animal Sciences, Zhejiang University, Hangzhou 310058, People's

* Correspondence: liujx@zju.edu.cn
Institute of Dairy Science, MoE Key Laboratory of Molecular Animal Nutrition,
College of Animal Sciences, Zhejiang University, Hangzhou 310058, People's

* Correspondence: liujx@zju.edu.cn
Institute of Dairy Science, MoE Key Laboratory of Molecular Animal Nutrition,
College of Animal Sciences, Zhejiang University, Hangzhou 310058, People's Republic of China
}

(c) 2016 The Author(s). Open Access This article is distributed under the terms of the Creative Commons Attribution 4.0 International License (http://creativecommons.org/licenses/by/4.0/), which permits unrestricted use, distribution, and reproduction in any medium, provided you give appropriate credit to the original author(s) and the source, provide a link to the Creative Commons license, and indicate if changes were made. The Creative Commons Public Domain Dedication waiver (http://creativecommons.org/publicdomain/zero/1.0/) applies to the data made available in this article, unless otherwise stated.

changes in diverse biological systems and may be the sole technology that can detect these changes [3]. This is further improved by combining nuclear magnetic resonance or mass spectra (MS) based high-throughput identification methods and multivariate statistical analyses [4]. The metabolomics approach is a useful tool for elucidating the effects of diet on biofluid metabolite profiles in dietary intervention studies on dairy cows [5].

In previous work, we characterized certain results for common metabolites in four biofluids (rumen fluid, milk, serum, and urine) [6]. We concluded that a deep 
analysis of metabolites from a single representative biofluid should be used to generate specific biomarkers and pathways to distinguish the metabolic profiles of dairy cows fed different diets. Urine metabolites result from global metabolism within the body and are easily affected by physiological, dietary and environmental interventions. As an easily collected and stored biofluid, urine has been widely used in human metabolomics to diagnose disease and serve as an early warning in preclinical stages [7]. Urine metabolomics may be used to capture most of the small molecular compounds in urine and identify those that significantly differ to further characterize metabolic pathways that may differ among different dietary treatments [8]. This will provide insight into identifying biomarkers and understanding the physiological processes associated with performance differences [9].

In the current study, biomarker and related pathway analysis was performed using the aforementioned urine metabolomics raw data under different forage treatments to evaluate the effects of forage quality on dairy cow metabolism. This approach can discern valid biomarkers and pathways and to understand the mechanism underlying forage-related nutrition in dairy cows.

\section{Methods}

\section{Experimental design}

The experimental procedures were approved by the Animal Care Committee at the Zhejiang University (Hangzhou, China) and were in accordance with the university's guidelines for animal research.

Sixteen multiparous (3.6 \pm 1.8 parity) Holstein cows were randomly assigned to 2 groups according to BW and milk yield as described by Sun et al. [6]. Both groups were offered consisting of $55 \%$ concentrate mixture and $15 \%$ corn silage with different forages (Additional file 1: Table S1): (1) alfalfa (AH), containing $23 \%$ alfalfa hay and $7 \%$ Chinese wild rye hay, and (2) corn stover (CS), containing $30 \%$ corn stover instead of alfalfa hay and Chinese wild rye hay. The diet was formulated to meet or exceed the net energy requirement for cows with milk production at $30 \mathrm{~kg} / \mathrm{d}$ [10]. Feed was offered ad libitum to allow for at least 5 to $10 \%$ orts. The experiment lasted for $65 \mathrm{~d}$ long with the first $15 \mathrm{~d}$ were used for adaption. Cows were housed in a tie-stall barn with free access to drinking water and were fed and milked 3 times daily at 0630,1330 and $1930 \mathrm{~h}$.

\section{Sample collection and metabolite measurement}

At the end of the experiment, urine samples $(15 \mathrm{~mL})$ were collected using vulval stimulation between 0500 and $0630 \mathrm{~h}$. Each sample was immediately frozen in a liquid nitrogen container to minimize metabolite degradation. After thawing and centrifugation at $6,000 \times \mathrm{g}$ at
$4{ }^{\circ} \mathrm{C}$ for $15 \mathrm{~min}$, samples were stored in $1.5 \mathrm{~mL}$ centrifuge tubes at $-80{ }^{\circ} \mathrm{C}$ for further analyses. The methods and procedures for identifying specific metabolomics, including metabolite extraction, derivatization, GC-TOF/ MS identification and data pretreatment, are described elsewhere [6]. L-2-chlorophenylalanine was used as a internal standard and bistrifluoroacetamide (containing $1 \%$ TCMS, v/v) as a derivated reagent. Agilent 7890 GC system equipped with a Pegasus 4D TOFMS (LECO, St. Joseph, MI, USA) was installed with a DB-5MS capillary column $(0.25 \mu \mathrm{m}$ film thickness, $30 \mathrm{~m} \times 250 \mu \mathrm{m}$ inner diameter). Helium served as the carrier gas and a front inlet purge flow of $3 \mathrm{~mL} / \mathrm{min}$ was utilized. Temperature procedure was as follows: the initial temperature was kept at $80{ }^{\circ} \mathrm{C}$ for $0.2 \mathrm{~min}$, increased to $180{ }^{\circ} \mathrm{C}$ at a rate of $10{ }^{\circ} \mathrm{C} / \mathrm{min}$, to $240{ }^{\circ} \mathrm{C}$ at a rate of $5{ }^{\circ} \mathrm{C} / \mathrm{min}$, and further to $290{ }^{\circ} \mathrm{C}$ at a rate of $20{ }^{\circ} \mathrm{C} / \mathrm{min}$; the column was then maintained for $11 \mathrm{~min}$. The injection, transfer line, and ion source temperatures were 280,245 , and $220{ }^{\circ} \mathrm{C}$, respectively. The MS data were acquired at a rate of 100 spectra/s after a solvent delay of $492 \mathrm{~s}$ with a mass-tocharge ratio $(\mathrm{m} / \mathrm{z})$ range of 20 to 600 in full-scan mode.

\section{Hierarchical cluster analysis}

In a previous study [6], 31 significantly different metabolites were identified in the urine. The relative concentration of these significantly different metabolites was incorporated into an online analysis platform (Metaboanalyst 3.0, http://www.metaboanalyst.ca/) for a hierarchical cluster analysis (HCA), which is a widely used data summary analysis tool to merge similar groups of points into visualization tree. Each sample began as a separate cluster, and the algorithm combined the samples until each sample belonged to one cluster. The HCA with Euclidean distance similarity measures and an average linkage method was used to explore clustering patterns among the samples and metabolites in urine. The expression patterns and a heat map of each variable were generated using an average linkage hierarchical clustering program. High-correlation samples were positioned near the top of the dendrogram, and highly similar metabolites were assigned near the left of the dendrogram. Other multivariate statistic analysis (principal component analysis (PCA) and partial least squares discriminant analysis (PLS-DA)) were also performed using Metabolyst 3.0. The PCA was used to visualize the dataset and display similarities and differences. PLS-DA was performed to sharpen the separation between groups of observations, and to understand which variables carry the class separating information.

\section{Biomarker analysis and validation}

Biomarker analysis was performed using statistical and mathematical modeling methods to select the minimum 
number of metabolites to represent and explain difference between 2 treatments. A subset of metabolites was manually selected to construct a classifier. One or more metabolites can be selected based on their difference between 2 groups (e.g., VIP value, $P$ value, fold change). The classifier was then evaluated as a biomarker by analyzing the receiver operating characteristic (ROC), including the ROC view, predicted class probabilities, and cross validation $(\mathrm{CV})$ prediction. The ROC analyses were based on a linear SVM algorithm. To produce a smooth ROC curve, 100 cross validations were performed, and results averaged to generate the plot. Using a probability view, a figure was generated to show the average predicted class probabilities for each sample among the 100 cross-validations. The classification boundary was located at the center for a balanced subsampling approach. Primarily, the effective sensitivity and specificity was evaluated based on the value of the area under the ROC curve (AUC), the sample distribution in the probability view, and $\mathrm{CV}$ prediction accuracy.

Biomarkers were validated by standard compound quantitative method using GC-TOF/MS. A standard curve was generated using a $4 \mathrm{mg} / \mathrm{mL}$ stock solution of hippuric acid (98 \% assay; Sgima-Aldrich). The equipment used is previously described. However the temperature procedure differed and as follows: the initial temperature was kept at $50{ }^{\circ} \mathrm{C}$ for $1 \mathrm{~min}$, then raised to $300{ }^{\circ} \mathrm{C}$ at a rate of $20{ }^{\circ} \mathrm{C} / \mathrm{min}$, then kept for $6.5 \mathrm{~min}$ at $300{ }^{\circ} \mathrm{C}$. The injection, transfer line, and ion source temperatures were 280,280 , and $220{ }^{\circ} \mathrm{C}$, respectively. The energy was $-70 \mathrm{eV}$ in electron impact mode. The mass spectrometry data were acquired in full-scan mode with the $\mathrm{m} / \mathrm{z}$ range of 30-600 at a rate of $20 \mathrm{spectra} / \mathrm{s}$ after a solvent delay of $4.93 \mathrm{~min}$.

\section{Related pathway characterization}

Pathway characterization is used to expand metabolomic analyses to understand the systems-level effects of

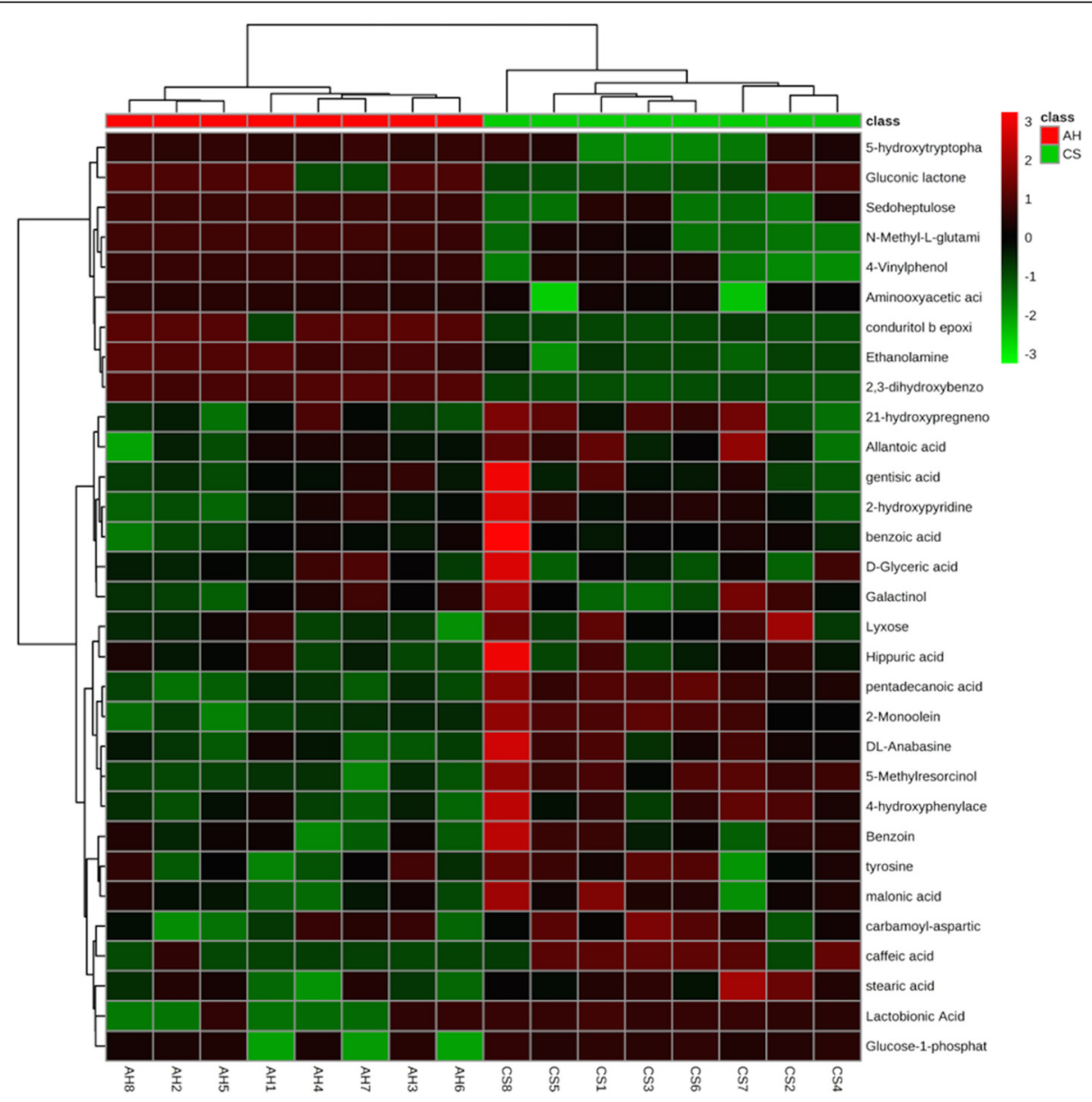

Fig. 1 A hierarchical clustering analysis for the significantly different urine metabolites. The patterns in each row were determined using an average linkage hierarchical clustering program. The light blue boxes indicate an expression ratio less than the mean, and the dark red boxes denote an expression ratio greater than the mean. Tree clusters and their shorter Euclidean distances indicate higher similarities. Similarity between two metabolites is represented by branch height; thus, when a node is lower vertically, the subtree is more similar 
metabolites [11]. The relative concentrations of 31 significantly different metabolites were imported into Metaboanalyst to generate the metabolome view, which integrates pathway enrichment analysis and pathway topology analysis. In doing so subtle but consistent changes among a group of related compounds may be identified [12]. A global test was used in pathway enrichment analysis to determine whether a group of metabolites in one specific pathway is differentially expressed, which shifts individual metabolite analysis to a group of metabolites analysis [13]. Pathway topological analysis was based on the relative betweenness and out of degree centrality measures of a metabolite in a given metabolic network to calculate the metabolites importance [14]. The pathway impact was calculated as the sum of the importance measures of the matched metabolites normalized by the importance of all metabolites in each pathway [15]. The differential response in metabolites between the two groups were further identified using online databases, including the Kyoto Encyclopedia of Genes and Genomes (KEGG), Bovine Metabolome Database, PubChem Compound, Chemical Entities of Biological Interest and Chemical Abstracts Service. Each different metabolite was crosslisted with the pathways in the KEGG; the top altered pathways were then identified and constructed according to the potential functional analysis.

\section{Results \\ Metabolic profiles and hierarchical cluster analysis}

The PCA and PLS-DA analyses of the GC-TOF/MS metabolic profiles clearly show separated clusters in the 2D-PCA score plot between the $\mathrm{AH}$ and $\mathrm{CS}$ groups (Additional file 1: Figure S1), suggesting that the GCTOF/MS-based urine metabolomics model can be used to identify the difference between the two diets.

The relative concentrations of 31 significantly different metabolites identified in the urine are shown in Fig. 1 with changes in the color intensity (from light blue to dark red) on the heat map. Different subclusters containing different numbers of samples in one diet fully clustered together, indicating a clear and strong difference between the AH and CS diets. Therefore, biomarker and pathway analyses based on these 31 significantly differently metabolites are credible.

\section{Analysis and validation of biomarker}

Based on the individual AUC, fold change (FC), and p-value, hippuric acid and N-methyl-L-glutamic acid (NML-Glu) were selected as potential biomarkers

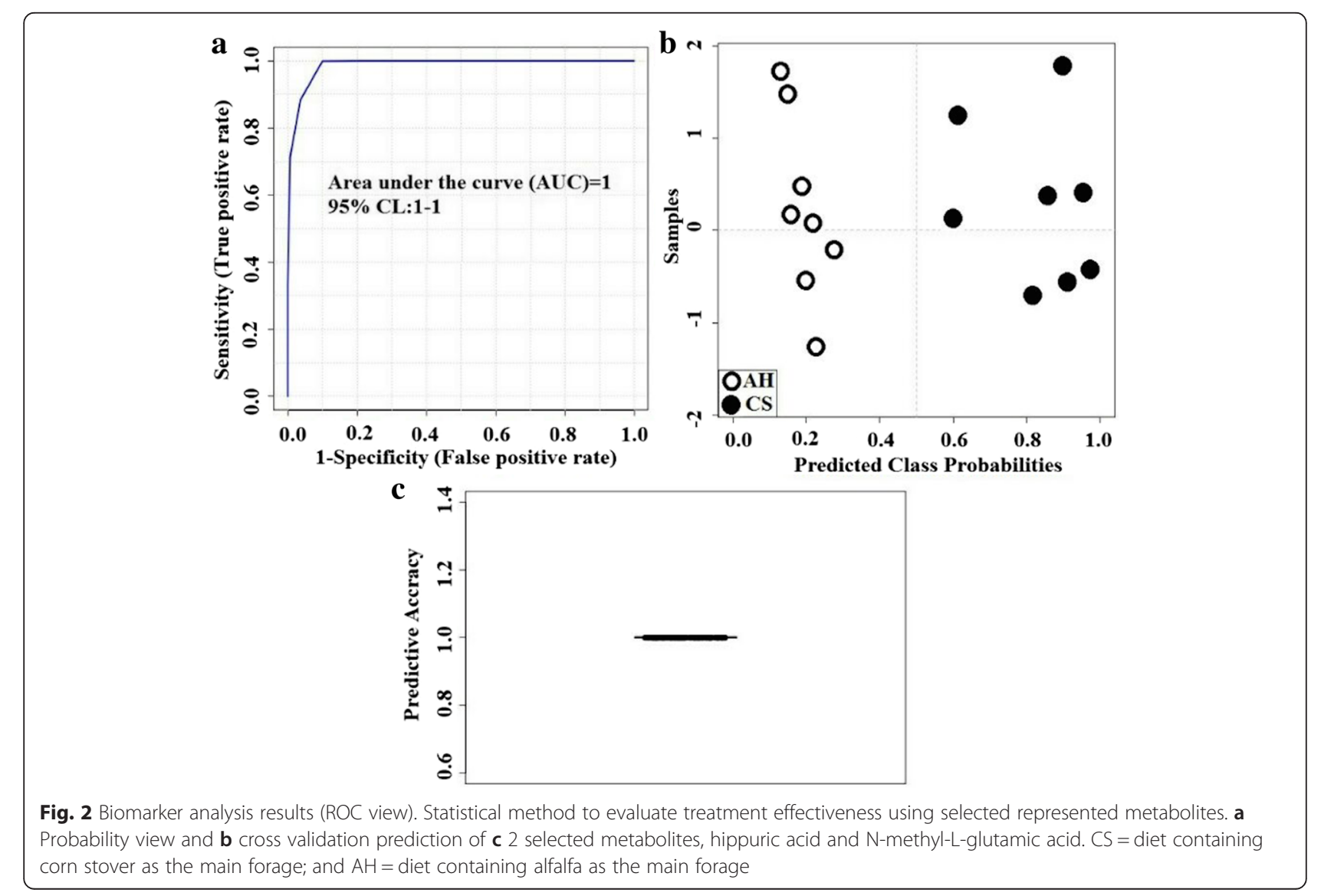


because they exhibited the most significant difference between the diets (Additional file 1: Table S1). The biomarker analysis results for HUA and NML-Glu are shown in Fig. 2. The AUC was equal to 1 (Fig. 2a), and a clear separation and discrimination were observed between the $\mathrm{CS}$ and $\mathrm{AH}$ diets in the probability view (Fig. 2b). An AUC close to 1 indicates a more effective sensitivity and specificity. The average accuracy based on 100 cross validations was 1 (Fig. 2c) in this study. Having an average accuracy close to 1 indicates a more valid $\mathrm{CV}$ prediction. The concentration of HUA $(\mathrm{mg} / \mathrm{mL})$ in CS group was significantly higher than that in $\mathrm{AH}$ group (2.8282 vs. $0.0005 ; P<0.01)$.

A significant negative correlation was observed between milk yield and HUA concentration $\left(R^{2}=0.459, P<0.01\right.$, Fig. 3a). On the contrary, milk yield was positively correlated with NML-Glu concentration $\left(R^{2}=0.652, P<0.01\right.$, Fig. $3 \mathrm{~b})$, suggesting that these 2 metabolites may serve as candidates for future investigations into forage-related lactation mechanisms.

\section{Characterization and functional analysis of key metabolic} pathways

Seventeen pathways were obtained when the significantly different metabolites were imported into KEGG. After enrichment and pathway topology analysis of the identified pathways, only 7 pathways showed an impact value at the comprehensive level (Table 1): Tyr metabolism; starch and sucrose metabolism; amino sugar and nucleotide sugar metabolism; galactose metabolism; Phe, Tyr and Try biosynthesis; purine metabolism; and glycerolipid metabolism. Among these 7 pathways, Phe, Tyr and Try biosynthesis exhibited the highest impact value (0.50). When the statistical $P$ values were further adjusted via the Holm-Bonferroni method for multiple testing, only the Tyr metabolism exhibited significant differences $(P=0.048$, Table 1$)$.

A comprehensive analysis of the $P$ value and impact value showed that the pathways that differed the most were Tyr metabolism and Phe, Tyr and Try biosynthesis (Fig. 4). The integrated key pathways and involved
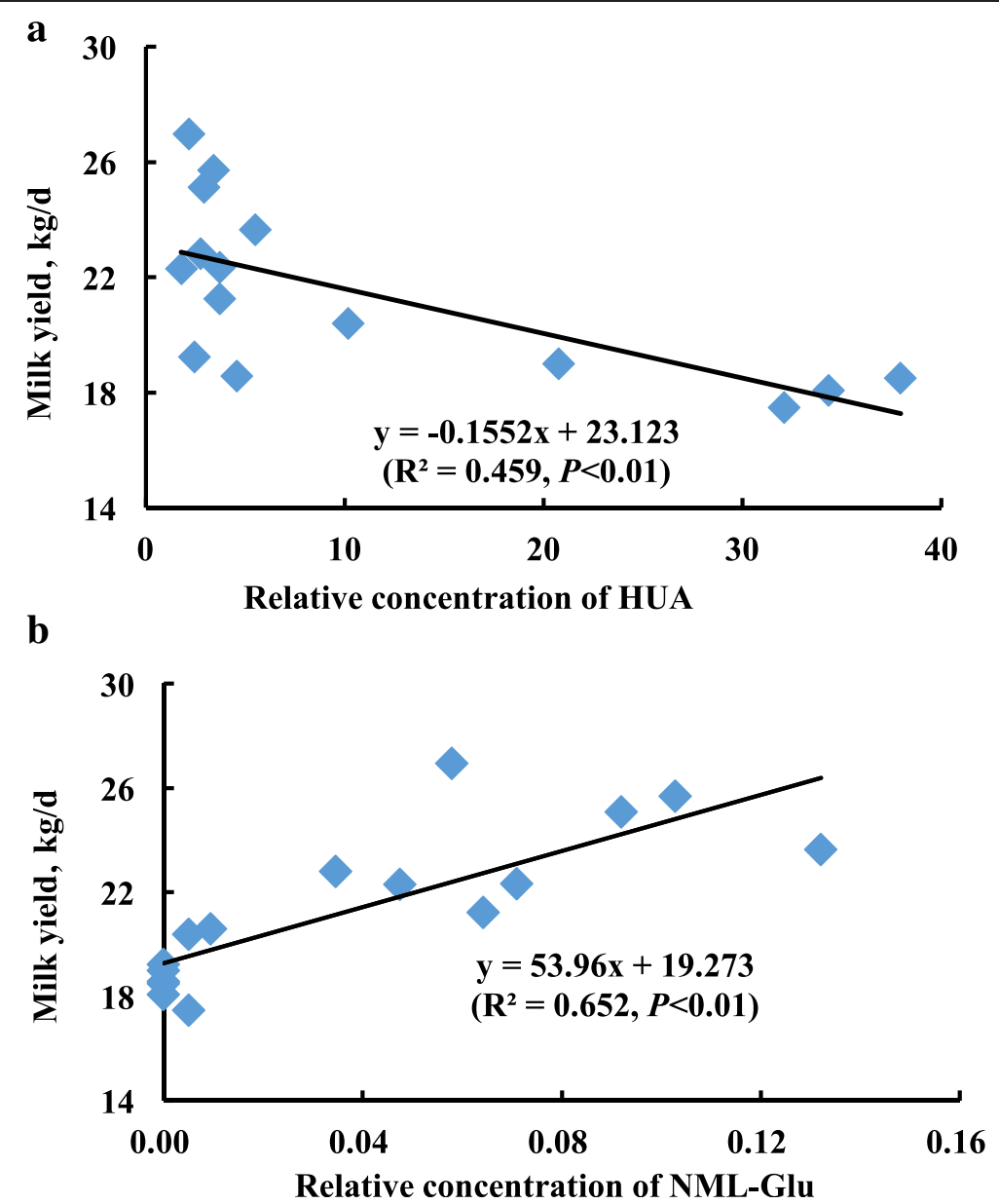

Fig. 3 Correlation of milk yield and relative concentrations of hippuric acid (HUA), and N-methyl-L-glutamic acid (NML-Glu) in lactating cows, 2 panels were combined together in one image 
Table 1 Results from the urine metabolomic pathway analyses in cows fed CS and AH diets

\begin{tabular}{llccc}
\hline Pathway & Hits $^{\mathrm{b}}$ & $P$ value & Home $P^{c}$ & Impact value \\
\hline Tyrosine metabolism & 3 & 0.003 & 0.048 & 0.145 \\
Starch and sucrose metabolism & 1 & 0.030 & 0.416 & 0.150 \\
Amino sugar and nucleotide sugar metabolism & 1 & 0.030 & 0.816 & 0.086 \\
Galactose metabolism & 2 & 0.097 & 1.000 & 0.119 \\
Phenylalanine, tyrosine and tryptophan biosynthesis & 1 & 0.138 & 1.000 & 0.500 \\
Purine metabolism & 1 & 0.152 & 1.000 & 0.0002 \\
Glycerolipid metabolism & 1 & 0.967 & & 0.105 \\
\hline
\end{tabular}

${ }^{\mathrm{a}} \mathrm{CS}=$ diet containing corn stover as the main forage; and $\mathrm{AH}=$ diet containing alfalfa hay as the main forage

${ }^{b}$ Hits represents the number of metabolites in one pathway

${ }^{C}$ Home $P$ indicates the statistical $P$ values that were further adjusted using the Holm-Bonferroni method for multiple tests

metabolites are shown in Fig. 5. For Tyr metabolism pathway, 3 significantly different metabolites were characterized: Tyr $(\mathrm{VIP}=1.38, \quad P=0.02 ; \quad \mathrm{FC}=2.84)$, 4Hydroxyphenylacetic acid (VIP $=1.35, P=0.03 ; \mathrm{FC}=$ 2.85 ) and gentisic acid (VIP $=1.21, P=0.04 ; \mathrm{FC}=0.47$ ). Tyr was also involved in Phe, Tyr and Try biosynthesis.

\section{Discussion}

The key biomarkers, HUA and NML-Glu were successfully investigated using GC-TOF/MS combined with biomarker analyses and statistically validated. This suggests that these 2 metabolites may be used as biomarkers in urine from dairy cows when corn stover replaces alfalfa hay.

The HUA is a potential marker for determining the best type of goat feeding regimen [16]. HUA is an acyl glycine formed from conjugating benzoic acid with glycine in the liver [17] and is one of the five major nitrogenous components in urine of dairy cows [18]. HUA excretion is related to dietary concentrations of degradable phenolic acids [19]. Forage contains high levels of aromatic compounds, including HUA [20], which can be partly degraded in the rumen. HUA is absorbed in the rumen and intestines following an immediate conjugation before being transformed in the liver and excreted in urine [21]. In the current study, significant higher amount of HUA in CS group was validated by both statistical analysis and standard compound quantitative method. The greater HUA excretion in the CS-fed cows partly resulted in greater nitrogen loss compared with the cows fed AH. HUA excretion has been linked to lignin digestibility [22]. Further work is necessary to validate HUA as a biomarker in dairy cow nutrition.

NML-Glu is a Glu derivative with a methyl group added to the amino group and is an intermediate of methane metabolism [23]. It can also regenerate Glu through methylglutamate dehydrogenase. Glu is the major milk protein component [24], accounting for more than $20 \%$ of milk protein and playing an important role in gluconeogenesis as a glucogenic precursor
[25]. The function of NML-Glu in the glutamate pool warrants further investigation.

Based on the KEGG pathways, Tyr metabolism was one of the 13 AA metabolism pathways (KEGG map 00350). As shown in Fig. 5, the altered Tyr metabolism in dairy cows fed different quality forage mainly resulted in Tyr degradation. In contrast, the alterations of Phe, Tyr and Try biosynthesis pathway was attributed to Tyr biosynthesis. Tyr is an aromatic AA and a precursor for adrenalin, dopamine, norepinephrine and epinephrine, which play an important role in the sympathetic nervous system in animals [26]. In general, Tyr concentration in blood depends on dietary Tyr content [27].

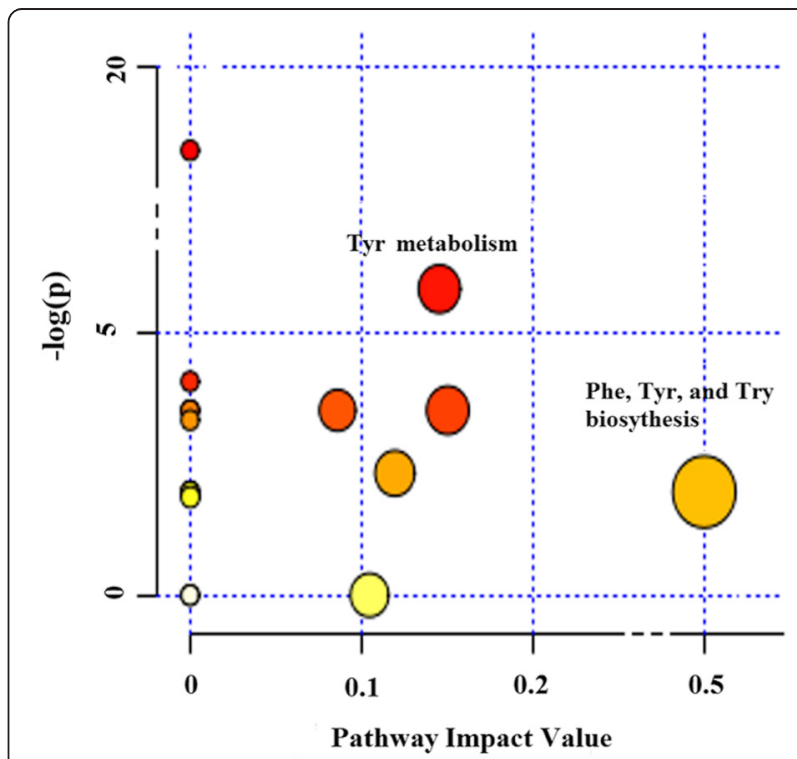

Fig. 4 The metabolome view map of significant metabolic pathways characterized in urine for cows fed CS and AH. This figure aims to find pathways significant changed based on enrichment and topology analysis. The $\mathrm{x}$-axis represents pathway enrichment, and the $y$-axis represents pathway impact. Larger sizes and darker colors represent greater pathway enrichment and higher pathway impact values, respectively. $\mathrm{CS}=$ diet containing corn stover as the main forage; and $\mathrm{AH}=$ diet containing alfalfa as the main forage 


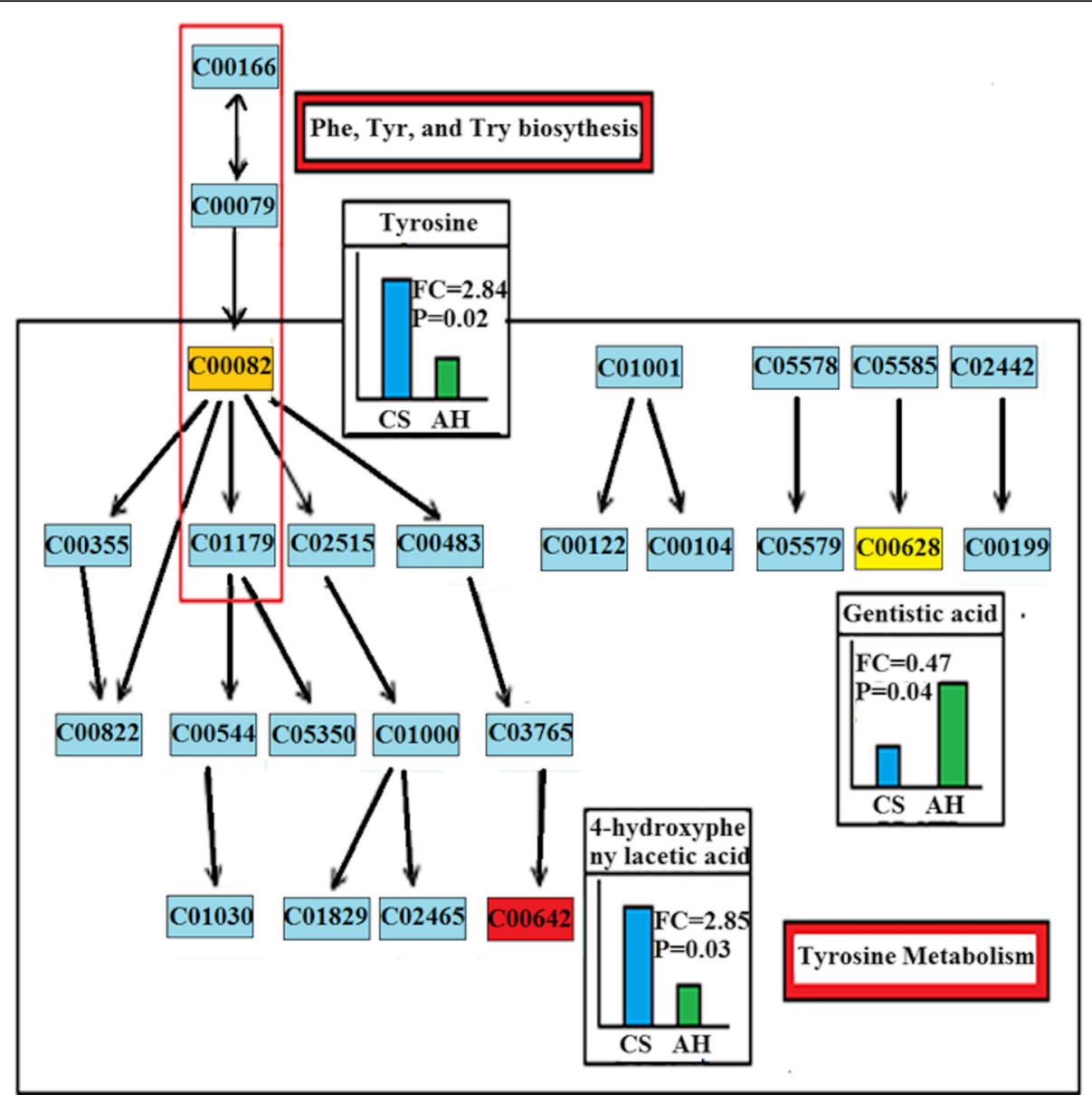

Fig. 5 Key pathways altered by different forage quality and associated metabolites. The map was generated using the reference map from KEGG and consisted of entry number of metabolites and pathways. Metabolites that significantly different are marked with yellow or red background and display with column figure of relative concentration between 2 diets. Common metabolites are marked with blue background. CO represents the entry number of the compound. $\mathrm{CS}=$ diet containing corn stover as the main forage; and $\mathrm{AH}=$ diet containing alfalfa as the main forage

Tyr is referred to as a semi-essential or conditionally indispensable AA because it only forms from Phe hydroxylation under certain condition [28]. During Tyr catabolism, the carboxyl carbon is almost immediately released as carbon dioxide, and the remaining portions of the Tyr molecule become either acetoacetate or fumarate [29], which can be used to synthesize AA or fatty acids.

Although Tyr is a non-essential AA, its synthesis within the body depends on Phe availability. Half of the Phe required for animals is used to produce Tyr. The requirement for Phe is reduced by approximately $50 \%$ with a Tyr-rich diet [30]. The same feature was also identified in a previous study on dairy cows [31]. Phe is mainly hydroxylated to Tyr in the hepatic intracellular pool, which is irreplaceable in Tyr biosynthesis. Phe is an essential AA that must be supplied by dietary proteins. Once it has entered the body, Phe may follow one of three pathways: conversion to Tyr, incorporation into cellular proteins, or conversion to phenylpyruvic acid [32]. For dairy cows, valine and other branched-chain AA may reduce tyrosine absorption [33], which may limit conversion of essential AA, such as Phe, into proteins.

The integrated metabolic pathways contain interaction networks as well as related metabolites and provide information on nutritional intervention mechanisms [34]. This information extends beyond metabolic relevance and effects through the pathways and network analyses applied in the metabolomics analyses [35]. The detailed construction of the altered Tyr metabolism; the Phe, Tyr and Try biosynthesis pathways map; and the related metabolites suggest that the target pathways yield marked changes when forage quality varies. These biochemical changes may be used to understand the effects of different quality forages on lactation performance and provide insight for future exploration of mechanisms and cow nutrition. 


\section{Conclusions}

GC-TOF/MS technology and multivariate analyses were used to to show significant changes in urine metabolites and metabolic pathways between two diets containing $\mathrm{AH}$ or CS as the main forage. The identified compounds hippuric acid and N-methy-l-Glu may serve as a potential biomarker for discriminating between different forage quality. The Tyr metabolism and Phe, Tyr and Try biosynthesis pathways showed the most variation when corn stover replaced alfalfa hay. Insight into foragerelated changes in physiology and metabolism may aid in developing strategies for better utilization of CS and other low-quality forages in China.

\section{Additional file}

Additional file 1: Table S1. Ingredients of the experimental diets based on corn stover and alfalfa hay. Table S2. Identification of significantly different metabolites in urine between the $\mathrm{CS}$ and AH groups. Figure S1. The 2-D PCA score map (a) and 2-D PLS-DA score map (b) derived from the GC-TOF/MS metabolite profiles of urine for cows fed CS (red triangle) and $\mathrm{AH}$ (green plus). $\mathrm{CS}=$ dietcontaining corn stover as main forage; and $\mathrm{AH}=$ dietcontaining alfalfa and Chinese wild rye hay as main forage. (DOC $145 \mathrm{~kb}$ )

\section{Abbreviations}

AH, Alfalfa hay; AUC, Area under the ROC curve; CS, Corn stover; CV, Cross validation; FC, Fold change; GC-TOF/MS, Gas chromatography-time of flight/ mass spectrometry; Glu, Glutamic acid; HCA, Hierarchical cluster analysis; HUA, Hippuric acid; KEGG, Kyoto encyclopedia of genes and genomes; MS, Mass spectra; PCA, Principal component analysis; Phe, Phenlalanine; PLS-DA, Partial least squares discriminant analysis; ROC, Receiver operating characteristic; SVM, Support vector machine; Try, Tryptophane; Tyr, Tyrosine; VIP, Variable importance projection.

\section{Acknowledgments}

The authors gratefully thank all of the staff of the Hangjiang Dairy Farm (Hangzhou, China) for their assistance in feeding, milking and care of the animals. We are grateful to Dr. Heather A. Tucker (Novus International Inc. St. Charles, US) for editing for grammar. We also acknowledge the members of the Institute of Dairy Science Zhejiang University for their assistance with urine sampling.

\section{Funding}

This research was supported by grants from the National Natural Science Foundation of China (No. 31472121) and from the China Agriculture Research System (No. CARS-37). The funding body has not participated in or interfered with the research.

\section{Availability of data and materials}

All data generated or analyzed during this study are included in this published article and its supplementary information files.

\section{Authors' contributions}

HZS designed the whole experiment, analyzed the data and wrote the manuscript. BW participated in experimental design, animal feeding trial, and sample measurements. JKW and HYL contributed to the manuscript revision. JXL was in charge of the whole trial. All authors read and approved the final manuscript.

\section{Competing interests}

The authors declare that they have no competing interests.

\section{Consent for publication}

Not applicable.

\section{Ethics approval and consent to participate}

The procedures of this study were approved by the Animal Care and Use Committee of Zhejiang University (Hangzhou, China) and were in accordance with the university's guidelines for animal research.

Received: 4 December 2015 Accepted: 10 August 2016

Published online: 31 August 2016

\section{References}

1. Zhu W, Fu Y, Wang B, Wang C, Ye JA, Wu YM, et al. Effects of dietary forage sources on rumen microbial protein synthesis and milk performance. J Dairy Sci. 2013;96(3):1727-34.

2. Wang B, Mao SY, Yang HJ, Wu YM, Wang JK, Li SL, et al. Effects of alfalfa and cereal straw as a forage source on nutrient digestibility and lactation performance in lactating dairy cows. J Dairy Sci. 2014;97(12):7706-15.

3. Zhang X, Shen J, Cao B, Xu L, Zhao T, Liu X, et al. Metabolomic investigation of Arthus reaction in a rat model using proton nuclear magnetic resonance (1 H NMR) spectroscopy and rapid resolution liquid chromatography (RRLC). Mol Biosyst. 2013;9(6):1423-35.

4. Zhao S, Zhao J, Bu D, Sun P, Wang J, Dong Z. Metabolomics analysis reveals large effect of roughage types on rumen microbial metabolic profile in dairy cows. Lett Appl Microbiol. 2014;59(1):79-85.

5. Saleem F, Ametaj BN, Bouatra S, Mandal R, Zebeli Q, Dunn SM. A metabolomics approach to uncover the effects of grain diets on rumen health in dairy cows. J Dairy Sci. 2012;95(11):6606-23.

6. Sun HZ, Wang DM, Wang B, Wang JK, Liu HY, Guan LL, et al. Metabolomics of four biofluids from dairy cows: potential biomarkers for milk production and quality. J Proteome Res. 2015;14:1287-98.

7. Wang X, Zhang A, Han Y, Wang P, Sun H, Song G, et al. Urine metabolomics analysis for biomarker discovery and detection of jaundice syndrome in patients with liver disease. Mol Cell Proteomics. 2012;11(8):370-80.

8. Zhang A, Sun H, Wu X, Wang X. Urine metabolomics. Clin Chim Acta. 2012; 414:65-9.

9. Bertram HC, Yde CC, Zhang X, Kristensen NB. Effect of dietary nitrogen content on the urine metabolite profile of dairy cows assessed by nuclear magnetic resonance (NMR)-based metabolomics. J Agri Food Chem. 2011; 59(23):12499-505.

10. Ministry of Agriculture, P.R.C. Feeding Standard of Dairy Cattle. Beijing: Academic; 2004. NY/T 34.

11. Johnson CH, Ivanisevic J, Siuzdak G. Metabolomics: beyond biomarkers and towards mechanisms. Nat Rev Mol Cell Bio. 2016;17:451-59.

12. Xia J, Sinelnikov I, Han B, Wishart DS. MetaboAnalyst 3.0 - making metabolomics more meaningful. Nucl Acids Res. 2015:43(W1):251-57.

13. Goeman JJ, Van De Geer SA, De Kort F, van Houwelingen HC. A global test for groups of genes: testing association with a clinical outcome. Bioinformatics. 2004;20(1):93-9.

14. Abbasi A, Hossain L, Leydesdorff L. Betweenness centrality as a driver of preferential attachment in the evolution of research collaboration networks. J Informetr. 2012;6(3):403-12

15. Xia J, Wishart DS. Met PA. A web-based metabolomics tool for pathway analysis and visualization. Bioinformatics. 2010;26(18):2342-44.

16. Carpio A, Bonilla-Valverde D, Arce C, Rodríguez-Estévez V, SánchezRodríguez M, Arce L. Evaluation of hippuric acid content in goat milk as a marker of feeding regimen. J Dairy Sci. 2013;96(9):5426-34.

17. Pero RW, Lund H. Dietary quinic acid supplied as the nutritional supplement $\mathrm{AlO}+\mathrm{AC}-11$ leads to induction of micromolar levels of nicotinamide and tryptophan in the urine. Phytother Res. 2011;25(6):851-57.

18. Dijkstra J, Oenema O, van Groenigen JW, Spek JW, van Vuuren AM, Bannink A. Diet effects on urine composition of cattle and $\mathrm{N}_{2} \mathrm{O}$ emissions. Animal. 2013;7(s2):292-302.

19. Spek JW, Dijkstra J, van Duinkerken G, Bannink A. A review of factors influencing milk urea concentration and its relationship with urinary urea excretion in lactating dairy cattle. J Agri Sci. 2012;151(03):407-23.

20. Gatley SJ, Sherratt HS. The synthesis of hippurate from benzoate and glycine by rat liver mitochondria. Submitochondrial localization and kinetics. Biochem J. 1977;166:39-47

21. Pero RW. Health consequences of catabolic synthesis of hippuric acid in humans. Curr Clin Pharmacol. 2010;5(1):67-73.

22. Kluge $\mathrm{H}$, Broz J, Eder K. Effects of dietary benzoic acid on urinary $\mathrm{pH}$ and nutrient digestibility in lactating sows. Livest Sci. 2010;134:119-21. 
23. Hao SL, Mata M, Wolfe D, Glorioso JC, Fink DJ. Gene transfer of glutamic acid decarboxylase reduces neuropathic pain. Ann Neurol. 2005;57:914-18.

24. Swaisgood HE. Protein and amino acid composition of bovine milk. In: Jensen RG, editor. Handbook of Milk Composition. San Diego: Academic; 1995. p. 464-71.

25. Treberg JR, Banh S, Pandey U, Weihrauch D. Intertissue differences for the role of glutamate dehydrogenase in metabolism. Neurochem Res. 2014; 39(3):516-26.

26. Sreekumar A, Poisson LM, Rajendiran TM, Khan AP, Cao Q, Yu J, et al. Metabolomic profiles delineate potential role for sarcosine in prostate cancer progression. Nature. 2009:457:910-14.

27. Matthews DE. An overview of phenylalanine and tyrosine kinetics in humans. J Nutr. 2007;137(6):1549-55.

28. Bequette BJ, Backwell FRC, Kyle CE, Calder AG, Buchan V, Crompton LA, et al. Vascular sources of phenylalanine, tyrosine, lysine, and methionine for casein synthesis in lactating goats. J Dairy Sci. 1999;82:362-77.

29. Gabr SA. Reproductive performance and milk yield of Friesian dairy cows affected by L-tyrosine treatment during early postpartum period. Life Sci J. 2012;9(4):4486-89.

30. Andrea GD, Ostuzzi R, Bolner A, Francesconi F, Musco F, Onofrio D, et al. Study of tyrosine metabolism in eating disorders. Possible correlation with migraine. Neurol Sci. 2008:29(1):88-92.

31. NRC. Nutrient Requirements of Dairy Cattle. 7th rev. ed. Natl. Acad. Sci., Washington, DC. 2001

32. Vockley J, Andersson HC, Antshel KM, Braverman NE, Burton BK, Frazier DM, et al. Phenylalanine hydroxylase deficiency: diagnosis and management guideline. Genet Med. 2014;16(2):188-200.

33. Crompton LA, France J, Reynolds CK, Mills JAN, Hanigan MD, Ellis JL, et al. An isotope dilution model for partitioning phenylalanine and tyrosine uptake by the mammary gland of lactating dairy cows. J Theor Bio. 2014; 359(21):54-60

34. Zhang GF, Sadhukhan S, Tochtrop GP, Brunengraber H. Metabolomics, pathway regulation, and pathway discovery. J Biol Chem. 2011;286:23631-35.

35. Creek DJ, Chokkathukalam A, Jankevics A, Burgess KE, Breitling R, Barrett MP. Stable isotope-assisted metabolomics for network-wide metabolic pathway elucidation. Anal Chem. 2012;84:8442-47.

\section{Submit your next manuscript to BioMed Central and we will help you at every step:}

- We accept pre-submission inquiries

- Our selector tool helps you to find the most relevant journal

- We provide round the clock customer support

- Convenient online submission

- Thorough peer review

- Inclusion in PubMed and all major indexing services

- Maximum visibility for your research

Submit your manuscript at www biomedcentral.com/submit

) Biomed Central 